\title{
DE L'AGRIPPEMENT SENSORIEL À LA MÉTAPHORE PARTAGÉE DANS LA CLINIQUE DE L'AUTISME
}

Chantal Lheureux-Davidse

\section{ERES | « Chimères »}

$2012 / 3 N^{\circ} 78$ | pages 75 à 85

ISSN 0986-6035

ISBN 9782749234649

Article disponible en ligne à l'adresse :

http://www.cairn.info/revue-chimeres-2012-3-page-75.htm

\section{Pour citer cet article :}

Chantal Lheureux-Davidse, « De l'agrippement sensoriel à la métaphore partagée dans la clinique de l'autisme », Chimères 2012/3 ( $\left.\mathrm{N}^{\circ} 78\right), \mathrm{p} .75-85$.

DOI 10.3917/chime.078.0075

Distribution électronique Cairn.info pour ERES.

(C) ERES. Tous droits réservés pour tous pays.

La reproduction ou représentation de cet article, notamment par photocopie, n'est autorisée que dans les limites des conditions générales d'utilisation du site ou, le cas échéant, des conditions générales de la licence souscrite par votre établissement. Toute autre reproduction ou représentation, en tout ou partie, sous quelque forme et de quelque manière que ce soit, est interdite sauf accord préalable et écrit de l'éditeur, en dehors des cas prévus par la législation en vigueur en France. Il est précisé que son stockage dans une base de données est également interdit. 


\section{CHANTAL LHEUREUX-DAVIDSE}

\section{De l'agrippement sensoriel à la métaphore partagée dans la clinique de l'autisme}

T A NÉCESSITÉ IMPÉRATIVE pour un enfant autiste de recourir à des Lagrippements sensoriels ou à des stéréotypies fait parfois effet de contrôle absolu. Elle donne le sentiment que toute communication est rompue car elle exclut la présence de l'autre. Ce système autoréféré qui impose un contrôle sur l'environnement fait penser parfois à une toute-puissance infantile où l'enfant tenterait de décider de tout dans la famille en inversant les rôles et les générations, comme cela arrive dans une étape du processus œedipien. Je montrerai en quoi ce contrôle dans les problématiques autistiques est spécifique et s'en différencie tant dans ses enjeux que dans ses manifestations. Dans l'autisme, l'agrippement sensoriel sur le corps propre ou l'environnement non humain peut être considéré comme une métaphore de la relation première. Je préciserai la place de l'autre dans la relation thérapeutique et son lien avec les éprouvés sensoriels.

En raison des difficultés d'accordage dans les relations premières, dans le regard et dans l'ajustement tonico-postural, le bébé se sent submergé par la relation en direct avec l'autre, car trop complexe et imprévisible. Il tente alors de contrôler ce qui n'est pas contrôlable, c'est-à-dire la relation à l'autre, ressentie comme une effraction. Il se met en retrait et déplace ses investissements sur l'environnement non humain qui est plus facilement contrôlable. La spécificité de

- Psychanalyste, maître de conférences à l'université Paris-Diderot. 
l'autisme concernant la toute-puissance infantile est que ce contrôle sur l'environnement s'exerce davantage sur l'environnement non humain que vis-à-vis de l'entourage familial. Il arrive cependant qu'un enfant autiste soit également tyrannique avec son entourage pour tenter de s'apaiser de saturations sensorielles insupportables tant que le démantèlement sensoriel ne lui permet pas d'intégrer plusieurs sensations à la fois. Je me souviens de Sami, jeune garçon autiste de douze ans qui reléguait sa mère à la cuisine pendant qu'il voulait regarder seul dans le salon une vidéo de son choix, en la passant à l'envers pour obtenir certaines sensations qui l'apaisaient.

La tentative de contrôle sur l'environnement d'un enfant autiste est bien différente de la toute-puissance infantile d'un enfant qui tenterait d'usurper la place du parent de sexe opposé comme dans le processus œdipien. Dans la clinique de l'autisme, comme chez Sami, nous nous situons bien avant le processus œedipien. Il s'agit davantage d'une protection contre un excès de sensations ou d'émotions qui déborde l'enfant au point qu'il préfere s'extraire provisoirement du lien en direct. En tentant de tout contrôler, et en excluant sa mère, Sami limite toute imprévisibilité qui procurerait chez lui un risque d'effraction catastrophique. Ce déplacement fait office d'exclusion des relations humaines en direct et peut faire effet de violence sur l'entourage familial qui se retrouve comme immobilisé et anéanti. Pour l'entourage, c'est la douleur éprouvée de se sentir inutile et même effractant pour un enfant qui est insupportable, comme si le retrait traduisait une indifférence qui effacerait le sens de la relation et de l'existence. Pour le thérapeute, penser l'évitement du lien direct en termes de débordement sensoriel au lieu d'y voir de l'indifférence donne de meilleures perspectives du côté d'une rencontre possible, même si la relation ne s'établit que par le détour de l'environnement non humain avant qu'une relation directe devienne envisageable.

\section{Nature des sensations recherchées dans les stéréotypies}

Si nous partons de l'a priori que les stéréotypies, les écholalies ou les comportements répétitifs d'une personne autiste lui sont nécessaires dans des moments de désorganisation pour se rassurer, nous pourrions alors nous intéresser à la nature des sensations obtenues. Nous constatons fréquemment que ces sensations recherchées font écho à 
celles que le bébé vit dans les relations premières, soit dans la vie intra-utérine soit très vite après la naissance. Par exemple en s'agrippant à une lumière trouvée dans l'environnement et en y adjoignant de petits mouvements devant les yeux avec une brindille ou en clignant des yeux en direction de la lumière, nous retrouvons des sensations que le bébé éprouve dans la rencontre avec le regard brillant de sa mère et en micro-accordage au moment où il est contenu dans l'attention maternelle.

Ces premières rencontres sont baignées dans une co-sensualité où sont convoqués le plaisir du contact corporel, l'échange visuel, la rencontre de l'odeur du bon lait, du parfum de la mère et celui du bébé, le plaisir gustatif de la tétée et un échange sonore de lallations ou de mots émotionnellement investis de part et d'autre. Chez les personnes autistes, c'est spécialement cette comodalité sensorielle qui pose problème dans l'ajustement relationnel.

\section{Saturation sensorielle et clivage}

En effet les troubles de l'intégration sensorielle sont fréquents chez les personnes autistes et chaque sens se trouve rapidement saturé entraînant parfois des brouillages sensoriels, comme a pu le décrire Temple Grandin, au point que toute sensation devient effractante. Pour remédier à ce chaos sensoriel, des installations en clivage deviennent alors nécessaires afin d'apaiser l'intensité ingérable de ces afflux sensoriels qui ne peuvent être encore filtrés en raison d'un défaut de constitution de pare-excitation.

Si l'installation en clivage est un recours salvateur pour apaiser le trop-plein de sensations, elle n'est possible qu'au prix d'une dispersion psychique qui rompt tous les liens logiques des sensations normalement reliées entre elles. En morcelant une grappe de sensations insupportables, chaque sensation devient indépendante des autres, moins intense et donc acceptable. Cette dispersion où chaque sens fonctionne pour lui-même empêche cependant tout relais d'une modalité sensorielle à une autre. C'est ainsi qu'une personne autiste se retrouve dans une incapacité provisoire à utiliser plusieurs sens à la fois pour traiter une information. Elle peut écouter mais ne peut pas voir en même temps, elle peut regarder mais sans pouvoir écouter ni comprendre ce qui est énoncé au même moment. Quand 
l'évitement du regard peut permettre à une personne autiste de mieux écouter, cela ne donne pas à l'entourage le sentiment d'être en communication. Le traitement de l'information reste partiel et les réactions sont souvent décalées, en différé et hors contexte.

\section{Interchangeabilité, confusion et effacement}

Ce morcellement de la compréhension d'une information entraîne souvent un état de chaos interne où chaque élément psychique devient interchangeable avec des risques de vécus confusionnels et le sentiment que des parties clivées sont anéanties. L'image du corps elle aussi est vécue comme morcelée et des vécus de disparition, d'effacement ou d'arrachement des extrémités du corps sont source d'angoisse. Par exemple la partie inférieure du corps peut être comme anéantie de l'image du corps et des vécus d'effondrement corporel peuvent devenir envahissants. Il arrive même que la personne s'effondre comme un chiffon au moindre changement imprévisible. La bouche est également une zone très fragile dans la constitution de l'image du corps. Cette zone, lorsqu'elle est déconstruite de l'image du corps, peut être vécue comme un trou béant à remplir ou à colmater pour éviter les angoisses de vidage ou d'effraction.

De même les enveloppes corporelles non constituées ne permettent pas de se sentir en sécurité. Didier Anzieu a parlé d'un moi-peau passoire. Tant que les parties du corps ne sont pas intégrées par la fonction de mise en réseau de la peau, les angoisses d'anéantissement, de dispersion ou d'effondrement sont fréquentes.

\section{Agrippement musculaire}

Pour lutter contre ces risques d'effondrement les personnes autistes mettent en place des stratégies inconscientes pour s'auto-maintenir. Par exemple des installations en carapace musculaire externe deviennent des équivalents d'une seconde peau selon l'expression d'Esther Bick, pour pallier à une première peau défaillante. Frances Tustin a bien décrit cet autisme à carapace. Certaines personnes autistes ont une démarche presque mécanique en raison de ce raidissement musculaire en carapace de leurs jambes, n'utilisant plus la souplesse des articulations des chevilles et des genoux jusqu'à marcher sur la pointe des pieds, tant que le bas du corps n'est pas intégré. Ces personnes ont d'ailleurs rarement investi le plaisir des arti- 
culations de leurs jambes quand ils étaient bébés et sont souvent passées de la position assise à la position debout sans explorer l'espace par la marche à quatre pattes. C'est parfois au cours de la thérapie qu'elles vont explorer ce mode de locomotion au moment de la découverte du plaisir des sensations éprouvées dans le bas de leur corps, comme Delphine, jeune fille autiste d'une quinzaine d'années, qui repartait joyeusement à quatre pattes en fin de séance au moment où elle retrouvait le plaisir dans ses jambes après avoir marché de nombreuses années sur la pointe des pieds dans un agrippement musculaire en carapace.

Parfois l'agrippement musculaire est interne et bloque la digestion ou la respiration. Il procure une sensation de solidité et de contrôle faute de sécurité interne fiable et en l'absence d'enveloppes psychiques constituées.

\section{Agrippements sensoriels et angoisses spatiales}

D'autres personnes en retrait autistique s'agrippent à une sensation trouvée en dehors de leur corps dans l'environnement non humain, comme par exemple lorsqu'elles s'accrochent à un contraste lumineux qu'elles tentent de réanimer avec une stéréotypie devant les yeux. Les bébés hospitalisés, lorsqu'ils sont privés de contact relationnel peuvent également s'agripper à un reflet trouvé sur le matériel de soin. Une stéréotypie peut s'adjoindre à l'agrippement sensoriel pour tenter d'animer le point lumineux ou le reflet en clignant des yeux par exemple comme pour le rendre vivant. Lorsque la stéréotypie est répétée, elle a un effet familier et rassurant. Elle sera convoquée à chaque moment difficile et servira à la personne autiste à se retrouver.

Cet agrippement sensoriel en adhésivité abrase toute notion de profondeur de l'espace. Si les angoisses spatiales sont trop fortes, la motricité peut se figer tout particulièrement lors du démarrage d'un mouvement pour tenter d'éviter des risques de vertiges dans l'espace. Une autre façon d'apaiser les angoisses spatiales consiste pour un enfant autiste à se coller à une paroi solide, comme à un mur en entrant dans une pièce. Il peut aussi tournoyer sur lui-même pour tenter de se recentrer en s'agrippant à son axe vertical. Quand le regard est retrouvé, il peut aussi s'allonger au sol, pour éprouver une 
sécurité d'arrière-plan dans son dos au contact avec cette surface dure qui le porte. Cela fait alors directement écho aux expériences premières avec le contenant utérin puis de portage du bébé dans les bras de sa mère tout particulièrement dans le contact de la nuque et du haut de la colonne vertébrale. Pour d'autres le recours à une stéréotypie pour explorer l'espace est nécessaire par exemple en agitant une brindille ou leurs doigts devant leurs yeux dans la direction de ce qu'ils regardent, pour combler le gouffre qui les sépare de ce qui est convoité par le regard. En conséquence de cette adhésivité qui pourtant les protège d'un risque d'effondrement catastrophique ou d'agonies primitives comme Winnicott a pu les décrire dans $\mathrm{La}$ crainte de l'effondrement, toute différenciation est suspendue et le processus d'individuation est mis à mal.

\section{Arrachement ou expérience de séparation : de l'auto-sensualité à l'auto-érotisme}

C'est la constitution des enveloppes psychiques personnelles qui prépare l'expérience de séparation sans que celle-ci soit vécue comme un arrachement. Nous remarquons souvent que le recours à ces mouvements répétitifs ou à ces stéréotypies est entretenu tant que les enveloppes psychiques ne sont pas assez sécures. Des moments de stéréotypies alternent avec des moments de tranquillité. Donald Meltzer nous a sensibilisés à la réversibilité du démantèlement sensoriel dans une alternance de démantèlement et de remantèlement. Cela se fait parallèlement avec la réversibilité de la construction de l'image du corps. Elle dépend des moments de dispersion psychique, d'expériences de désorganisation ou des moments de sécurité.

Un agrippement sensoriel est parfois convoqué dans l'urgence comme une bouée de sauvetage dans un instant de survie psychique face à une expérience de désorganisation et ne peut être considéré comme de l'auto-érotisme. En effet l'auto-érotisme suppose la notion d'altérité qui est comme gommée dans ces moments. Il s'agit plutôt d'auto-sensualité où seule la sensation obtenue pour se sentir exister est recherchée à chaque instant. Mais une absence de contenant psychique stable empêche la mobilisation des traces mnésiques. Chaque sensation doit alors être répétée pour être retrouvée à chaque instant et les instants ne semblent reliés entre eux que par la 
rythmicité de l'entretien des stéréotypies pour éviter les ruptures catastrophiques à chaque changement imprévisible. Ce système rythmique auto-entretenu constitue une sorte de contrôle sur l'environnement nécessaire pour éviter l'effondrement et pour entretenir le sentiment d'exister.

\section{De l'agrippement sensoriel à la métaphore partagée}

Dans d'autres cas l'agrippement sensoriel dans l'environnement non humain ou sur son propre corps permet de retrouver un repère familier auto-généré et produit un confort rassurant qui favorise un rassemblement après un moment de dispersion. Mais de quelles sensations s'agit-il dans ces agrippements et stéréotypies ? L'observation clinique nous montre qu'elles sont en lien avec les sensations corporelles normalement éprouvées quand l'image du corps se constitue et avec celles qui sont obtenues généralement dans la première rencontre avec l'autre. Une lumière normalement trouvée dans le regard maternel associée aux micromouvements d'accordage dans l'échange des regards sera ici recherchée dans des sensations lumineuses animées contrôlées, auto-entretenues. Elles permettent d'éviter toute imprévisibilité d'un regard qui serait vécu comme engloutissant. Mais elles en sont également des métaphores par déplacement.

Il faut le passage de l'autre pour que ces agrippements sensoriels soient repérés comme des équivalents métaphoriques des sensations trouvées dans la relation première mère-bébé ou pendant les étapes de construction de l'image du corps. Par exemple, il n'est pas rare d'observer que des personnes présentant des troubles de l'image du corps se balancent d'avant en arrière de façon rythmique. C'est le cas de nombreuses personnes autistes et nous retrouvons ce type de balancement également chez des personnes souffrant de psychose. En observant davantage, nous nous apercevons que ces balancements se font fréquemment à la fréquence d'un battement par seconde, ce qui correspond au rythme cardiaque maternel. D'autres balancements plus lents font étrangement écho au rythme respiratoire maternel. Ce sont deux rythmes dans lesquels toute personne a été baignée in utero lorsqu'elle était un foetus, qui sont recherchés pour obtenir un apaisement.

Comment l'agrippement sensoriel peut-il se transformer en une rencontre avec l'autre? 
Si nous supposons que ces sensations recherchées de façon autistique sont une parcelle d'éprouvé d'une rencontre mère-bébé, nous pouvons envisager que le travail du thérapeute serait de rendre partageables ces instants d'agrippement sensoriel en les considérant comme nécessaires dans un premier temps. Cet a priori me paraît être un préalable pour entrer en contact avec une personne autiste. De même nous pourrions dire que l'a priori que tout est langage chez un bébé est un préalable pour lui parler. Il pourrait en être de même avec des personnes autistes. Parfois l'imitation spontanée sert de miroir et de reconnaissance aux sensations éprouvées. Mais il est plus naturel d'entrer en contact avec un bébé en lui parlant que de s'émerveiller sur une stéréotypie en la commentant, d'autant plus si la personne autiste est déjà grande dans son corps et qu'elle évite la relation directe en se réfugiant dans une lumière trouvée dans son environnement. Cependant ce détour par l'environnement non humain inanimé est une sorte de garantie pour la personne autiste qu'un contrôle reste possible avant qu'une relation de confiance ne s'établisse.

Ce mode de relation par un détour nous oblige à élargir notre conscience au-delà d'une identification moïque, c'est-à-dire en nous représentant que nos capacités d'identifications ne se limitent pas au monde humain. C'est à mon sens dans ces sphères que naviguent les personnes autistes. Et c'est ainsi que ces personnes se trouvent tellement isolées et si peu comprises. Notre difficulté à nous identifier au monde non humain renforce leur retrait. C'est par le biais des sensations trouvées dans l'environnement non humain mais éprouvées corporellement que cela devient possible.

Les poètes, les artistes, les personnes créatives côtoient ce mode de pensée qui est plus associatif que logique et qui fonctionne par effet de résonance bien au-delà d'une référence corporelle moïque ou contextuelle. Une rencontre devient possible sur ces plans métaphoriques. C'est par une attention bienveillante du thérapeute à ces particularités sensorielles et gestuelles, par des commentaires sur les sensations éprouvées ou par l'imitation gestuelle et par la présence respectueuse parfois silencieuse dans un premier temps, que la personne autiste peut s'y retrouver par effet de résonance. Elle peut enfin se sentir l'auteur de ses sensations qui étaient pour la plupart senties inconsciemment, bien qu'activement recherchées. 


\section{Jubilation partagée}

Une expérience de stéréotypie qui ne tient pas compte de l'altérité reste dans une recherche purement sensorielle sans que l'on puisse parler d'auto-érotisme comme nous l'avons évoqué précédemment. Quand un thérapeute est là pour reconnaître le plaisir retrouvé dans cette auto-sensualité, ses commentaires transforment les sensations inconscientes en perception et elles peuvent passer alors dans un registre auto-érotique. Car ce passage dans le registre perceptif est une garantie pour se sentir l'auteur de ses sensations afin de se sentir exister dans la présence d'un autre. C'est la jubilation de se sentir exister à la suite de commentaires identifiants qui procure un mouvement spontané vers un désir de partage émotionnel avec le thérapeute. La reconnaissance par l'autre du plaisir sensoriel éprouvé transforme une auto-sensualité en auto-érotisme.

Les sensations devenues conscientes par le passage d'un autre font miroir et donnent accès au sentiment d'en être l'auteur et d'accéder à la perception, ce qui est source d'un moment de sentiment d'exister. Généralement, les retrouvailles avec son sentiment d'exister provoquent un sursaut tonique, l'enfant se redresse dans le haut de son corps, et il manifeste une jubilation à l'origine d'une recherche de partage émotionnel. Alors la recherche de la source sonore de la voix à l'origine de ces commentaires permet de découvrir l'autre en direct souvent de façon fugitive et renforce ce sentiment d'exister mais cette fois-ci dans un échange avec un autre. C'est cette découverte de l'autre qui tisse des moments de rencontre créative au rythme de la personne qui peut ainsi sortir un moment de son retrait.

La souplesse entre des échanges sur des plans métaphoriques et ces instants de rencontres en direct favorisent l'intégration d'instants partagés intenses dans cette vie de solitaire. Les témoignages de Birger Sellin concernant son enfermement autistique en sont l'illustration lorsqu'il parle dans ses ouvrages de sa "solitude du déserteur " et de son " âme prisonnière ". Ainsi, c'est paradoxalement à partir de ces moments d'isolement les plus forts que la rencontre devient possible si le thérapeute peut se contenter dans un premier temps de rencontres élargies sur le plan des métaphores au-delà du registre strictement humain et moïque, même si l'objectif est d'apprivoiser le lien par ce détour afin qu'un cumul d'expériences en 
direct se constitue par la suite. Par ailleurs les réponses des personnes autistes sont rarement immédiates et il est fréquent que les réponses aux sollicitations lorsqu'elles deviennent possibles se fassent en différé, le temps que la personne se sente concernée et réalise sa place dans les rencontres. De plus la focalisation sur des détails se fait au détriment d'une vue d'ensemble, d'après les travaux de Laurent Mottron. La personne autiste peut passer en revue toutes les expériences vécues en lien associatif avec une question posée avant de pouvoir s'exprimer. Cette focalisation sur des détails pourrait être comprise comme un agrippement sensoriel pour éviter l'effondrement. Cette hypothèse semble être juste quand nous observons des personnes autistes de haut niveau dessinant détail après détail par contiguïté plutôt que dans un va-et-vient entre détails et vue d'ensemble.

D'autre part, la pensée est tellement solitaire chez une personne en retrait, que le registre de l'expression des pensées ou des émotions n'est généralement pas encore cultivé. C'est ainsi que les réponses données sont très parcellaires et ne passent dans un premier temps ni par les expressions faciales, qui sont pendant tout un temps encore gelées, ni par le langage verbal.

La moindre manifestation fait alors office de réponse et il appartient au thérapeute de repérer si ces réponses concernent le registre de la relation et de la reconstruction de l'image du corps ou bien alors si ces bribes de réponses traduisent une désorganisation, un refus ou une effraction.

Il est important de respecter le rythme de croissance psychique de la personne qui est souvent lent et d'aménager un espace transitionnel nécessaire à une intersubjectivité retrouvée. Pour que les personnes autistes se sentent concernées par les commentaires que nous leur proposons, ceux-ci gagneraient à être très ancrés dans la sensorialité des mots ou dans la référence aux sensations éprouvées. En se sentant concernée, la personne autiste retrouve à ce moment-là le sentiment d'exister, jubile et éprouve spontanément un désir de partage émotionnel qui passe par une rencontre dans les regards. Ce qui concourt à l'édifice de la construction du lien à l'autre. 


\section{Bibliographie}

Anzieu, D. 1987. "Les signifiants formels et le moi-peau ", Les Enveloppes psychiques, Paris, Dunod, p. 1-22.

Bick, E. 1968. "The Experiences of the Skin in early Object-relations ", int. Journal of Psychoanalyse, 49, 184-6, trad. fr. G. et M. Haag, "L'expérience de la peau dans les relations d'objets précoces ", in Meltzer D., et coll., Explorations dans le monde de l'autisme, 1975, p. 240-244.

Grandin, T. 1997. Penser en images et autres témoignages sur l'autisme, Paris, Odile Jacob, 261 p.

Haag, G., "Sexualité orale et moi corporel ", Topique, L'Esprit du temps, n 87, 2004/2, p. 23-45.

Lheureux-Davidse, C. 2003. L'autisme infantile ou le bruit de la rencontre. Contribution à une clinique des processus thérapeutiques, Paris, L'Harmattan, 342 p.

Lheureux-Davidse, C. 2007. "Jouer avec les mouvements, les vibrations et les rythmes dans l'émergence de la voix ", Champs psychosomatiques, La Voix, $\mathrm{n}^{\circ} 48$, Paris, L'Esprit du temps, p. 185-203.

Maiello, S. 2000. Trames sonores et rythmiques primordiales. Réminiscences auditives dans le travail psychanalytique, in " la croissance psychique ", Journal de la psychanalyse de l'enfant, $\mathrm{n}^{\circ}$ 26, Bayard Éditions, juin, p. 77-104.

Meltzer, D., Williams, M. H. 2000. L'appréhension de la beauté. Le conflit esthétique, son rôle dans le développement, la violence, l'art, Larmor-Place, éditions du Hublot.

Nadel, J., Decety, J. 2002. Imiter pour découvrir l'humain. Psychologie, neurobiologie, robotique et philosophie de l'esprit, Paris, PUF.

Racamier, P.-C. 1992. Le génie des origines. Psychanalyse et psychoses, Paris, Payot, coll. "Bibliothèque scientifique ", 420 p.

Searles, H. 1986, The nonhuman Environment, trad. fr. D. Blanchard, L'environnement non humain, Nrf, Gallimard, 358 p.

Sellin, B. 1994. Une âme prisonnière. Grâce à la communication facilitée, un jeune autiste nous révèle son univers, Éd. Robert Laffont, coll. "Réponses ".

Trevarthen, C., "Racines du langage avant la parole ", Devenir, vol.9, n 3, p. 73-93.

Tustin, F. 1986. The protective Shell in Children and Adults, London, Karnac Books, trad. fr., Autisme et protection, Paris, Seuil.

Winnicott, D. 1975. The Fear of Breakdown, trad. J. Kalmanovitch et M. Gribinski, "La crainte de l'effondrement ", Figures du vide, Nouvelle Revue de Psychanalyse, Paris, Nrf, Gallimard, 1975, 11, p. 35-44. 